\title{
Interpreting connexive principles in coherence-based probability logic
}

\author{
Niki Pfeifer ${ }^{1 \star \star \star}$ and Giuseppe Sanfilippo ${ }^{2 \star \star \star \star}$ \\ 1 Department of Philosophy, University of Regensburg, Germany \\ niki.pfeifer@ur.de \\ 2 Department of Mathematics and Computer Science, University of Palermo, Italy \\ giuseppe.sanfilippo@unipa.it
}

\begin{abstract}
We present probabilistic approaches to check the validity of selected connexive principles within the setting of coherence. Connexive logics emerged from the intuition that conditionals of the form If $\sim A$, then $A$, should not hold, since the conditional's antecedent $\sim A$ contradicts its consequent $A$. Our approach covers this intuition by observing that for an event $\mathrm{A}$ the only coherent probability assessment on the conditional event $A \mid \bar{A}$ is $p(A \mid \bar{A})=0$. Moreover, connexive logics aim to capture the intuition that conditionals should express some "connection" between the antecedent and the consequent or, in terms of inferences, validity should require some connection between the premise set and the conclusion. This intuition is covered by a number of principles, a selection of which we analyze in our contribution. We present two approaches to connexivity within coherence-based probability logic. Specifically, we analyze connections between antecedents and consequents firstly, in terms of probabilistic constraints on conditional events (in the sense of defaults, or negated defaults) and secondly, in terms of constraints on compounds of conditionals and iterated conditionals. After developing different notions of negations and notions of validity, we analyze the following connexive principles within both approaches: Aristotle's Theses, Aristotle's Second Thesis, Abelard's First Principle and selected versions of Boethius' Theses. We conclude by remarking that coherence-based probability logic offers a rich language to investigate the validity of various connexive principles.
\end{abstract}

Keywords: Aristotle's Theses · Coherence - Compounds of conditionals - Conditional events · Conditional random quantities · Connexive logic . Iterated conditionals · Probabilistic constraints.

\section{Introduction}

We present probabilistic approaches to check the validity of selected connexive principles within the setting of coherence. Connexive logics emerged from the

\footnotetext{
* Both authors contributed equally to the article and are listed alphabetically.

$\star \star$ Supported by the BMBF project 01UL1906X.

$\star \star \star$ Member of the GNAMPA Research Group and partially supported by the INdAM-GNAMPA Project 2020 Grant U-UFMBAZ-2020-000819.
} 
intuition that conditionals of the form if not- $A$, then $A$, denoted by $\sim A \rightarrow A$, should not hold, since the conditional's antecedent not- $A$ contradicts its consequent $A$. Indeed, experimental psychological data show that people believe that sentences of the form if not- $A$, then $A$ are false (e.g., 39 40]), which supports the psychological plausibility of this intuition. Connexive principles were developed to rule out such self-contradictory conditionals (for overviews, see e.g., 36153). Many of these principles can be traced back to antiquity or the middle ages, which is reflected by the names of these principles, for example, Aristotle's Thesis or Abelard's First Principle (see Table 1). In classical logic,

\begin{tabular}{lll}
\hline Name & \multicolumn{2}{l}{ Abbreviation Connexive principle } \\
\hline Aristotle's Thesis & (AT) & $\sim(\sim A \rightarrow A)$ \\
Aristotle's Thesis' & $\left(\mathrm{AT}^{\prime}\right)$ & $\sim(A \rightarrow \sim A)$ \\
Abelard's First Principle & (AB) & $\sim((A \rightarrow B) \wedge(A \rightarrow \sim B))$ \\
Aristotle's Second Thesis & (AS) & $\sim((A \rightarrow B) \wedge(\sim A \rightarrow B))$ \\
Boethius' Thesis & $(\mathrm{BT})$ & $(A \rightarrow B) \rightarrow \sim(A \rightarrow \sim B)$ \\
Boethius' Thesis' & $\left(\mathrm{BT}^{\prime}\right)$ & $(A \rightarrow \sim B) \rightarrow \sim(A \rightarrow B)$ \\
Reversed Boethius' Thesis & $\left(\mathrm{RBT}^{\prime}\right)$ & $\sim(A \rightarrow \sim B) \rightarrow(A \rightarrow B)$ \\
Reversed Boethius' Thesis' & $\left(\mathrm{RBT}^{\prime}\right)$ & $\sim(A \rightarrow B) \rightarrow(A \rightarrow \sim B)$ \\
Boethius Variation 3 & $(\mathrm{B} 3)$ & $(A \rightarrow B) \rightarrow \sim(\sim A \rightarrow B)$ \\
Boethius Variation 4 & (B4) & $(\sim A \rightarrow B) \rightarrow \sim(A \rightarrow B)$ \\
\hline \multicolumn{2}{c}{ Table 1: Selected connexive principles (see also $[53])}$.
\end{tabular}

however, Aristotle's Thesis, i.e. $\sim(\sim A \rightarrow A)$, is not a theorem since the corresponding material conditional is contingent because $\sim(\sim \sim A \vee A)$ is logically equivalent to $\sim A$ (which is not necessarily true). Moreover, connexive logics aim to capture the intuition that conditionals should express some "connection" between the antecedent and the consequent or, in terms of inferences, validity should require some connection between the premise set and the conclusion.

The connexive intuition that conditionals of the form if not- $A$, then $A$ should not hold is covered in subjective probability theory. Specifically, we cover this intuition by the observation that for any event $A$, with $\bar{A} \neq \varnothing$, the only coherent assessment on the conditional event $A \mid \bar{A}$ is $p(A \mid \bar{A})=0$.

The aim of our contribution is to investigate selected connexive principles within the framework of coherence-based probability logic. The coherence approach to (subjective) probability was originated by Bruno de Finetti (see, e.g., [1112]) and has been generalised to the conditional probability and to previsions of conditional random quantities (see, e.g., 2|3|6|9|21|24|29|33|49|52]). In the present framework, we present two approaches to connexivity within coherencebased probability logic. In the first approach we analyze connections between antecedents and consequents in terms of probabilistic constraints on conditional events (in the sense of defaults or negated defaults [16|44|45|46]). In the second approach, based the recently developed more general framework of compounds of conditionals and iterated conditionals ([18|19|22|24|27]), we define these connections in terms of constraints on suitable conditional random quantities. After 
developing different notions of negations and notions of validity, we analyze the connexive principles given in Table 1 within both approaches.

The coherence principle plays a key role in probabilistic reasoning and allows for probabilistic inferences of a further conditional event (the conclusion) from any coherent probabilistic assessment on an arbitrary family of conditional events (the premises). Moreover, coherence is a more general approach to conditional probabilities compared to approaches which requires positive probability for the conditioning events. In standard approaches to probability the conditional probability $p(C \mid A)$ is defined by the ratio $p(A \wedge C) / p(A)$, which requires positive probability of the conditioning event, $p(A)>0$. However, in the framework of coherence, conditional probability $p(C \mid A)$, as a degree of belief, is a primitive notion and it is properly defined even if the conditioning event has probability zero, i.e., $p(A)=0$. Analogously, within coherence, previsions of conditional random quantities, are primitive and properly defined even if the conditioning event has probability zero. Therefore, coherence is a more general approach to conditional probabilities compared to approaches which requires positive probability for the conditioning events. The only requirement is that the conditioning event must be logically possible. Thus, although $p(C \mid A)$ is well defined even if $p(A)=0$, it is undefined if $A \equiv \varnothing$ (where $\varnothing$ denotes a logical contradiction). This is in line with the reading that Boethius and Aristotle thought that principles like (BT) and (AT), respectively, hold only when the conditional's antecedent is possible (see 34] who argues that the "ancient logicians most likely meant their theses as applicable only to 'normal' conditionals with antecedents which are not selfcontradictory"; p. 16). It is also in line with the Ramsey test, which is expressed in his famous footnote: "If two people are arguing 'If $A$ will $C$ ?' and are both in doubt as to $A$, they are adding $A$ hypothetically to their stock of knowledge and arguing on that basis about $C$; so that in a sense 'If $A, C$ ' and 'If $A, \bar{C}$ ', are contradictories. We can say they are fixing their degrees of belief in $C$ given $A$. If $A$ turns out false, these degrees of belief are rendered void" [48, p. 155, we adjusted the notation]. The quantitative interpretation of the Ramsey test became a cornerstone of the conditional probability interpretation of conditionals. Adding a contradiction to your stock of knowledge does not make sense (as, traditionally, knowledge implies truth). Moreover, Ramsey's thought that conditionals with contradicting consequents $C$ and $\bar{C}$ contradict each other coincides with the underlying intuition of $(\mathrm{AB})$.

\section{Preliminary Notions and Results}

Given two events $A$ and $H$, with $H \neq \varnothing$ (where $\varnothing$ denotes the impossible event), the conditional event $A \mid H$ (read: $A$ given $H$ ) is defined as a three-valued logical entity which is true if $A H$ (i.e., $A \wedge H$ ) is true, false if $\bar{A} H$ is true, and void if $H$ is false. We observe that $A \mid H$ assumes the logical value (true or false) of $A$, when $H$ is true, and it is void, otherwise. There is a long history of how to deal with negations (see, e.g., [30]). In our context, the negation of the conditional event " $A$ given $H$ ", denoted by $\overline{A \mid H}$, is the conditional event $\bar{A} \mid H$, 
that is "the negation of $A$ " given $H$. We use the inner negation to preserve for conditional events the usual property of negating unconditional events: $p(\bar{A})=$ $1-p(A)$. In the subjective approach to probability based on the betting scheme, a conditional probability assessment $p(A \mid H)=x$ means that, for every real number $s$, you are willing to pay an amount $s \cdot x$ and to receive $s$, or 0 , or $s \cdot x$ (money back), according to whether $A H$ is true, or $\bar{A} H$ is true, or $\bar{H}$ is true (bet called off), respectively. The random gain, which is the difference between the (random) amount that you receive and the amount that you pay, is $G=(s A H+0 \bar{A} H+s x \bar{H})-s x=s A H+s x(1-H)-s x=s H(A-x)$.

Given a probability function $p$ defined on an arbitrary family $\mathcal{K}$ of conditional events, consider a finite subfamily $\mathcal{F}=\left\{A_{1}\left|H_{1}, \ldots, A_{n}\right| H_{n}\right\} \subseteq \mathcal{K}$ and the vector $\mathcal{P}=\left(x_{1}, \ldots, x_{n}\right)$, where $x_{i}=p\left(A_{i} \mid H_{i}\right)$ is the assessed probability for the conditional event $A_{i} \mid H_{i}, i=1, \ldots, n$. With the pair $(\mathcal{F}, \mathcal{P})$ we associate the random gain $G=\sum_{i=1}^{n} s_{i} H_{i}\left(A_{i}-x_{i}\right)$. We denote by $\mathcal{G}_{\mathcal{H}_{n}}$ the set of values of $G$ restricted to $\mathcal{H}_{n}=H_{1} \vee \cdots \vee H_{n}$, i.e., the set of values of $G$ when $\mathcal{H}_{n}$ is true. Then, we recall below the notion of coherence in the context of the betting scheme.

Definition 1. The function $p$ defined on $\mathcal{K}$ is coherent if and only if, $\forall n \geqslant 1$, $\forall s_{1}, \ldots, s_{n}, \forall \mathcal{F}=\left\{A_{1}\left|H_{1}, \ldots, A_{n}\right| H_{n}\right\} \subseteq \mathcal{K}$, it holds that: $\min \mathcal{G}_{\mathcal{H}_{n}} \leqslant 0 \leqslant$ $\max \mathcal{G}_{\mathcal{H}_{n}}$.

In betting terms, the coherence of conditional probability assessments means that in any finite combination of $n$ bets, after discarding the case where all the bets are called off, the values of the random gain are neither all positive nor all negative (i.e., no Dutch Book). In particular, coherence of $x=p(A \mid H)$ is defined by the condition $\min \mathcal{G}_{H} \leqslant 0 \leqslant \max \mathcal{G}_{H}, \forall s$, where $\mathcal{G}_{H}$ is the set of values of $G$ restricted to $H$ (that is when the bet is not called off). Depending on the logical relations between $A$ and $H$ (with $H \neq \varnothing$ ), the set $\Pi$ of all coherent conditional probability assessments $x=p(A \mid H)$ is:

$$
\Pi=\left\{\begin{array}{l}
{[0,1], \text { if } \varnothing \neq A H \neq H} \\
\{0\}, \quad \text { if } A H=\varnothing \\
\{1\}, \quad \text { if } A H=H
\end{array}\right.
$$

In numerical terms, once $x=p(A \mid H)$ is assessed by the betting scheme, the indicator of $A \mid H$, denoted by the same symbol, is defined as 1 , or 0 , or $x$, according to whether $A H$ is true, or $\bar{A} H$ is true, or $\bar{H}$ is true. Then, by setting $p(A \mid H)=x$,

$$
A \mid H=A H+x \bar{H}=\left\{\begin{array}{l}
1, \text { if } A H \text { is true } \\
0, \text { if } \bar{A} H \text { is true, } \\
x, \text { if } \bar{H} \text { is true. }
\end{array}\right.
$$

Note that since the three-valued numerical entity $A \mid H$ is defined by the betting scheme once the value $x=p(A \mid H)$ is assessed, the definition of (the indicator of) $A \mid H$ is not circular. The third value of the random quantity $A \mid H$ (subjectively) depends on the assessed probability $p(A \mid H)=x$. Moreover, the value $x$ coincides with the corresponding conditional prevision, denoted by $\mathbb{P}(A \mid H)$, 
because $\mathbb{P}(A \mid H)=\mathbb{P}(A H+x \bar{H})=p(A H)+x p(\bar{H})=p(A \mid H) p(H)+x p(\bar{H})=$ $x p(H)+x p(\bar{H})=x$.

In the special case where $A H=H$, it follows by (11) that $x=1$ is the only coherent assessment for $p(A \mid H)$; then, for the indicator $A \mid H$ it holds that

$$
A \mid H=A H+x \bar{H}=H+\bar{H}=1, \quad \text { if } A H=H .
$$

In particular (3) holds when $A=\Omega$ (i.e., the sure event), since $\Omega \wedge H=H$ and hence $\Omega|H=H| H=1$. Likewise, if $A H=\varnothing$, it follows by (11) that $x=0$ is the only coherent assessment for $p(A \mid H)$; then,

$$
A \mid H=0+0 \bar{H}=0, \quad \text { if } A H=\varnothing .
$$

In particular (4) holds when $A=\varnothing$, since $\varnothing \wedge H=\varnothing$ and hence $\varnothing \mid H=0$. We observe that conditionally on $H$ be true, for the (indicator of the) negation it holds that $\overline{A \mid H}=\bar{A}=1-A=1-A \mid H$. Conditionally on $H$ be false, by coherence, it holds that $\overline{A \mid H}=p(\bar{A} \mid H)=1-p(A \mid H)=1-A \mid H$. Thus, in all cases it holds that

$$
\overline{A \mid H}=\bar{A}|H=(1-A)| H=1-A \mid H .
$$

We denote by $X$ a random quantity, with a finite set of possible values. Given any event $H \neq \varnothing$, agreeing to the betting metaphor, if you assess the prevision $\mathbb{P}(X \mid H)=\mu$ means that for any given real number $s$ you are willing to pay an amount $s \mu$ and to receive $s X$, or $s \mu$, according to whether $H$ is true, or false (bet called off), respectively. In particular, when $X$ is (the indicator of) an event $A$, then $\mathbb{P}(X \mid H)=P(A \mid H)$. The notion of coherence can be generalized to the case of prevision assessments on a family of conditional random quantities (see, e.g., [25151]). Given a random quantity $X$ and an event $H \neq \varnothing$, with prevision $\mathbb{P}(X \mid H)=\mu$, likewise formula (2) for the indicator of a conditional event, an extended notion of a conditional random quantity, denoted by the same symbol $X \mid H$, is defined as follows $X \mid H=X H+\mu \bar{H}$. We recall now the notion of conjunction of two (or more) conditional events within the framework of conditional random quantities in the setting of coherence (1922 24|26], for alternative approaches see also, e.g., 31|37). Given a coherent probability assessment $(x, y)$ on $\{A|H, B| K\}$, we consider the random quantity $A H B K+x \bar{H} B K+y \bar{K} A H$ and we set $\mathbb{P}[(A H B K+x \bar{H} B K+y \bar{K} A H) \mid(H \vee K)]=z$. Then we define the conjunction $(A \mid H) \wedge(B \mid K)$ as follows:

Definition 2. Given a coherent prevision assessment $p(A \mid H)=x, p(B \mid K)=y$, and $\mathbb{P}[(A H B K+x \bar{H} B K+y \bar{K} A H) \mid(H \vee K)]=z$, the conjunction $(A \mid H) \wedge(B \mid K)$ is the conditional random quantity defined as

$$
\begin{aligned}
& (A \mid H) \wedge(B \mid K)=(A H B K+x \bar{H} B K+y \bar{K} A H) \mid(H \vee K)= \\
& =\left\{\begin{array}{l}
1, \text { if } A H B K \text { is true } \\
0, \text { if } \bar{A} H \vee \bar{B} K \text { is true, } \\
x, \text { if } \bar{H} B K \text { is true, } \\
y, \text { if } A H \bar{K} \text { is true, } \\
z, \text { if } \bar{H} \bar{K} \text { is true. }
\end{array}\right.
\end{aligned}
$$


Of course, $\mathbb{P}[(A \mid H) \wedge(B \mid K)]=z$. Coherence requires that the FréchetHoeffding bounds for prevision of the conjunction are preserved (22]), i.e, $\max \{x+y-1,0\} \leqslant z \leqslant \min \{x, y\}$, like in the case of unconditional events. Other preserved properties are listed in 27. We notice that if conjunctions of conditional events are defined as suitable conditional events (see, e.g., [1/5/7/8|28]), classical probabilistic properties are not preserved. In particular, the lower and upper probability bounds for the conjunction do not coincide with the above mentioned Fréchet-Hoeffding bounds ([50]). Here, differently from conditional events which are three-valued objects, the conjunction $(A \mid H) \wedge(B \mid K)$ is not any longer three-valued object, but a five-valued object with values in $[0,1]$. We observe that $(A \mid H) \wedge(A \mid H)=A \mid H$ and $(A \mid H) \wedge(B \mid K)=(B \mid K) \wedge(A \mid H)$. Moreover, if $H=K$, then $(A \mid H) \wedge(B \mid H)=A B \mid H$.

For comparison with other approaches, like [13, see [25, Section 9].

In analogy to formula (2), where the indicator of a conditional event " $A$ given $H$ " is defined as $A \mid H=A \wedge H+p(A \mid H) \bar{H}$, the iterated conditional " $B \mid K$ given $A \mid H "$ is defined as follows (see, e.g., [18|19|22]):

Definition 3 (Iterated conditioning). Given any pair of conditional events $A \mid H$ and $B \mid K$, with $A H \neq \varnothing$, the iterated conditional $(B \mid K) \mid(A \mid H)$ is defined as the conditional random quantity $(B \mid K)|(A \mid H)=(A \mid H) \wedge(B \mid K)+\mu \bar{A}| H$, where $\mu=\mathbb{P}[(B \mid K) \mid(A \mid H)]$.

Notice that we assume $A H \neq \varnothing$ to avoid trivial cases of iterated conditionals. Specifically, similar as in the three-valued notion of a conditional event $A \mid H$ where the antecedent $H$ must not be impossible (i.e., $H$ must not coincide with the constant 0$)$, the iterated conditional $(B \mid K) \mid(A \mid H)$ requires that the antecedent $A \mid H$ must not be constant and equal to 0 (this happens when $A H \neq \varnothing)$. Furthermore, we recall that the compound prevision theorem is preserved, that is $\mathbb{P}[(A \mid H) \wedge(B \mid K)]=\mathbb{P}[(B \mid K) \mid(A \mid H)] p(A \mid H)$.

\section{Approach 1: Connexive Principles and Default Reasoning}

In order to validate the connexive principles we interpret a conditional $A \rightarrow C$ by the default $A \sim C$, where $A$ and $C$ are two events (with $A \neq \varnothing$ ). A default $A \sim C$ can be read as $C$ is a plausible consequence of $A$ and is interpreted by the probability constraint $p(C \mid A)=1([16]) 3$ The conditional $A \rightarrow \sim C$ is interpreted by the default $A \sim \bar{C}$. Likewise, $\sim A \rightarrow C$ is interpreted by $\bar{A} \sim C$. A negated conditional $\sim(A \rightarrow C)$ is interpreted by the negated default $\sim(A \sim C)$

\footnotetext{
${ }^{3}$ According to $\varepsilon$-semantics (see, e.g., 1138) a default $A \sim C$ is interpreted by $p(C \mid A) \geqslant 1-\varepsilon$, with $\varepsilon>0$ and $p(A)>0$. Gilio introduced a coherence-based probability semantics for defaults by also allowing $\varepsilon$ and $p(A)$ to be zero ([15]). In this context, defaults in terms of probability 1 can be used to give a alternative definition of p-entailment which preserve the usual non-monotonic inference rules like those of System P (415]2021], see also 910]). For the psychological plausibility of the coherence-based semantics of non-monotonic reasoning, see, e.g., 41|42 |43|47].
} 
(it is not the case that: $C$ is a plausible consequence of $A$; also denoted by $A \not \psi C$ in [14|32]) that is $p(C \mid A) \neq 1$, which corresponds to the wide scope negation of negating conditionals ([16]).

The conjunction of two conditionals, denoted by $(A \rightarrow B) \wedge(C \rightarrow D)$, is interpreted by the sequence of their associated defaults $(A \sim B, C \sim D)$, which represents in probabilistic terms the constraint $(p(B \mid A)=1, p(D \mid C)=$ $1)$, that is $(p(B \mid A), p(D \mid C))=(1,1)$. Then, the negation of the conjunction of two conditionals, denoted by $\sim((A \rightarrow B) \wedge(C \rightarrow D))$ (i.e., in terms of defaults $\sim(A \sim B) \wedge(C \sim D))$ is interpreted by the negation of the probabilistic constraint $(p(B \mid A), p(D \mid C))=(1,1)$, that is $(p(B \mid A), p(D \mid C)) \neq(1,1)$. Table 2 summarizes the interpretations.

We now introduce the definition of validity for non-iterated connexive principles (e.g., (AT), $\left.\left(\mathrm{AT}^{\prime}\right),(\mathrm{AB})\right)$.

Definition 4. We say that a non-iterated connexive principle is valid if and only if the probabilistic constraint associated with the connexive principle is satisfied by every coherent assessment on the involved conditional events.

In the next paragraphs we check the validity in terms of Definition 4 of the non-iterated connexive principles in Table 1.

Aristotle's Thesis $(A T): \sim(\sim A \rightarrow A)$. We assume that $\bar{A} \neq \varnothing$ and we interpret the principle $\sim(\sim A \rightarrow A)$ by the negated default $\sim(\bar{A} \sim A)$ with the following associated probabilistic constraint: $p(A \mid \bar{A}) \neq 1$. We observe that $p(\bar{A} \mid A)=0$ is the unique precise coherent assessment on $\bar{A} \mid A$. Then, (AT) is valid because every coherent precise assessment $p(A \mid \bar{A})$ is such that $p(A \mid \bar{A}) \neq 1$.

Aristotle's Thesis' $\left(A T^{\prime}\right): \sim(A \rightarrow \sim A)$. Like (AT), $(\mathrm{AT})^{\prime}$ can be validated.

Abelard's Thesis $(A B): \sim((A \rightarrow B) \wedge(A \rightarrow \sim B))$. We assume that $A \neq \varnothing$. The structure of this principle is formalized by $\sim((A \sim B) \wedge(A \sim \bar{B}))$ which expresses the constraint $(p(B \mid A), p(\bar{B} \mid A)) \neq(1,1)$. We recall that coherence requires $p(B \mid A)+p(\bar{B} \mid A)=1$. Then, (AT) is valid because each coherent assessment on $(B|A, \bar{B}| A)$ is necessarily of the form $(x, 1-x)$, with $x \in[0,1]$, which of course satisfies $(p(B \mid A), p(\bar{B} \mid A)) \neq(1,1)$.

Aristotle's Second Thesis $(A S): \sim((A \rightarrow B) \wedge(\sim A \rightarrow B))$. We assume that $A \neq \varnothing$ and $\bar{A} \neq \varnothing$. The structure of this principle is formalized by $\sim((A \sim$ $B) \wedge(\bar{A} \sim B))$ which expresses the constraint $(p(B \mid A), p(B \mid \bar{A})) \neq(1,1)$. We recall that, given two logically independent events $A$ and $B$ every assessment $(x, y) \in[0,1]^{2}$ on $(B|A, B| \bar{A})$ is coherent. In particular, $(p(B \mid A), p(B \mid \bar{A}))=$ $(1,1)$ is a coherent assessment which does not satisfy the probabilistic constraint $(p(B \mid A), p(B \mid \bar{A})) \neq(1,1)$. Thus, (AS) is not valid.

Concerning iterated connexive principles (e.g. $(\mathrm{BT}),\left(\mathrm{BT}^{\prime}\right)$ ), we interpret the main connective $(\rightarrow)$ as the implication $(\Rightarrow)$ from the probabilistic constraint on the antecedent to the probabilistic constraint on the conclusion. Then, for instance, the iterated conditional $(A \rightarrow B) \rightarrow(C \rightarrow D)$ is interpreted by the implication $A \sim B \Rightarrow C \sim D$, that is $p(B \mid A)=1 \Rightarrow p(D \mid C)=1$. We now define validity for iterated connexive principles. 
Definition 5. An iterated connexive principle $\bigcirc \Rightarrow \square$ is valid if and only if the probabilistic constraint of the conclusion $\square$ is satisfied by every coherent extension to the conclusion from any coherent probability assessment satisfying the constraint of the premise $\bigcirc$.

\begin{tabular}{ccc}
\hline Conditional object & Default & Probabilistic interpretation \\
\hline$A \rightarrow C$ & $A \sim C$ & $p(C \mid A)=1$ \\
$\sim(A \rightarrow C)$ & $\sim(A \sim C)$ & $p(C \mid A) \neq 1$ \\
$(A \rightarrow B) \wedge(C \rightarrow D)$ & $(A \sim B, C \sim D)$ & $(p(B \mid A), p(D \mid C))=(1,1)$ \\
$\sim((A \rightarrow B) \wedge(C \rightarrow D))$ & $\sim(A \sim B, C \sim D)$ & $(p(B \mid A), p(D \mid C)) \neq(1,1)$ \\
$(A \rightarrow B) \rightarrow(C \rightarrow D)$ & $A \sim B \Rightarrow C \sim D$ & $p(B \mid A)=1 \Rightarrow p(D \mid C)=1$ \\
\hline
\end{tabular}

Table 2: Probabilistic interpretations of logical operation on conditionals in terms of defaults or negated defaults.

We check the validity in terms of Definition 5 of the iterated connexive principles in Table 1

Boethius' Thesis (BT): $(A \rightarrow B) \rightarrow \sim(A \rightarrow \sim B)$. We assume that $A \neq \varnothing$. This is interpreted by the implication $A \sim B \Rightarrow \sim(A \sim \bar{B})$, that is $p(B \mid A)=1 \Rightarrow p(\bar{B} \mid A) \neq 1$. We observe that, by setting $p(B \mid A)=1$, $p(\bar{B} \mid A)=1-p(B \mid A)=0$ is the unique coherent extension to $\bar{B} \mid A$. Then, as $p(B \mid A)=1 \Rightarrow p(\bar{B} \mid A)=0 \neq 1$, the iterated connexive principle (BT) is valid.

Boethius' Thesis' $\left(B T^{\prime}\right):(A \rightarrow \sim B) \rightarrow \sim(A \rightarrow B)$. Like $(\mathrm{BT})$, it can be shown that $\left(\mathrm{BT}^{\prime}\right)$ is valid too.

Reversed Boethius' Thesis (RBT): $\sim(A \rightarrow \sim B) \rightarrow(A \rightarrow B)$. We assume that $A \neq \varnothing$. This is interpreted by $\sim(A \sim \bar{B}) \Rightarrow A \sim B$, that is $p(\bar{B} \mid A) \neq 1 \Rightarrow p(B \mid A)=1$. We observe that, by setting $p(\bar{B} \mid A)=x$ it holds that $p(B \mid A)=1-x$ is the unique coherent extension to $B \mid A$. In particular by choosing $x \in] 0,1[$, it holds that $p(\bar{B} \mid A) \neq 1$ and $p(B \mid A) \neq 1$. Thus, $p(\bar{B} \mid A) \neq 1 \nRightarrow p(B \mid A)=1$ and hence (RBT) is not valid.

Reversed Boethius' Thesis' $\left(R B T^{\prime}\right): \sim(A \rightarrow B) \rightarrow(A \rightarrow \sim B)$. Like (RBT), it can be shown that $\left(\mathrm{RBT}^{\prime}\right)$ is not valid too.

Boethius Variation (B3): $(A \rightarrow B) \rightarrow \sim(\sim A \rightarrow B)$. We assume that $A \neq \varnothing$ and $\bar{A} \neq \varnothing$. This is interpreted by $A \sim B \Rightarrow \sim(\bar{A} \sim B)$, that is $p(B \mid A)=1 \Rightarrow p(B \mid \bar{A}) \neq 1$. We observe that, by setting $p(B \mid A)=1$, any value $p(B \mid \bar{A}) \in[0,1]$ is a coherent extension to $B \mid \bar{A}$, because the assessment $(1, y)$ on $(B|A, B| \bar{A})$ is coherent for every $y \in[0,1]$. In particular the assessment $(1,1)$ on $(B|A, B| \bar{A})$ is coherent. Therefore, as $p(B \mid A)=1 \nRightarrow p(B \mid \bar{A}) \neq 1$, (B3) is not valid.

Boethius Variation (B4): $(\sim A \rightarrow B) \rightarrow \sim(A \rightarrow B)$. Like (B3), it can be shown that (B4) is not valid too.

We summarize the results of this section in Table 3 


\begin{tabular}{ccccc}
\hline Name & Connexive principle & Default & Probabilistic constraint & Validity \\
\hline (AT) & $\sim(\sim A \rightarrow A)$ & $\sim(\bar{A} \sim A)$ & $p(A \mid \bar{A}) \neq 1$ & yes \\
(AT') & $\sim(A \rightarrow \sim A)$ & $\sim(A \sim \bar{A})$ & $p(\bar{A} \mid A) \neq 1$ & yes \\
(AB) & $\sim((A \rightarrow B) \wedge(A \rightarrow \sim B))$ & $\sim(A \mid \sim B, A \sim \bar{B})$ & $(p(B \mid A), p(\bar{B} \mid A)) \neq(1,1)$ & yes \\
(AS) & $\sim((A \rightarrow B) \wedge(\sim A \rightarrow B))$ & $\sim(A \mid \sim B, \bar{A} \sim B)$ & $(p(B \mid A), p(B \mid \bar{A})) \neq(1,1)$ & no \\
(BT) & $(A \rightarrow B) \rightarrow \sim(A \rightarrow \sim B)$ & $A \sim B \Rightarrow \sim(A \mid \sim \bar{B})$ & $p(B \mid A)=1 \Rightarrow p(\bar{B} \mid A) \neq 1$ & yes \\
(BT') & $(A \rightarrow \sim B) \rightarrow \sim(A \rightarrow B)$ & $A \sim \bar{B} \Rightarrow \sim(A \sim B)$ & $p(\bar{B} \mid A)=1 \Rightarrow p(B \mid A) \neq 1$ & yes \\
(RBT) & $\sim(A \rightarrow \sim B) \rightarrow(A \rightarrow B)$ & $\sim(A \sim \bar{B}) \Rightarrow A \sim B$ & $p(\bar{B} \mid A) \neq 1 \Rightarrow p(B \mid A)=1$ & no \\
(RBT') & $\sim(A \rightarrow B) \rightarrow(A \rightarrow \sim B)$ & $\sim(A \sim B) \Rightarrow A \mid \sim \bar{B}$ & $p(B \mid A) \neq 1 \Rightarrow p(\bar{B} \mid A)=1$ & no \\
(B3) & $(A \rightarrow B) \rightarrow \sim(\sim A \rightarrow B)$ & $A \sim B \Rightarrow \sim(\bar{A} \sim \sim B)$ & $p(B \mid A)=1 \Rightarrow p(B \mid \bar{A}) \neq 1$ & no \\
(B4) & $(\sim A \rightarrow B) \rightarrow \sim(A \rightarrow B)$ & $\bar{A} \sim B \Rightarrow \sim(A \mid \sim B)$ & $p(B \mid \bar{A})=1 \Rightarrow p(B \mid A) \neq 1$ & no
\end{tabular}

Table 3: Connexive principles in the framework of defaults and probabilistic constraints (Approach 1).

\section{Approach 2: Connexive Principles and Compounds of Conditionals}

In this section we analyze connexive principles within the theory of logical operations among conditional events. Specifically, we analyze connections between antecedents and consequents in terms of constraints on compounds of conditionals and iterated conditionals. In this second approach, a basic conditional $A \rightarrow C$ is interpreted as a conditional event $C \mid A$ (instead of a probabilistic constraint on conditional events) which is a three-valued object: $C \mid A \in\{1,0, x\}$, where $x=p(C \mid A)$. The negation $\sim(A \rightarrow C)$ is interpreted by $\bar{C} \mid A$ (which is the narrow scope negation of negating conditionals). Then, $\sim(A \rightarrow \sim C)$ amounts to $\bar{C} \mid A$ which coincides with $C \mid A$. We recall that logical operations among conditional events do not yield a conditional event, rather they yield conditional random quantities with more than three possible values (see, e.g., 22]). Then, we interpret the results of the logical operations in the connexive principles by suitable conditional random quantities. In particular, the conjunction $(A \rightarrow B) \wedge(C \rightarrow D)$ (resp., $\sim((A \rightarrow B) \wedge(C \rightarrow D)))$ is interpreted by $(B \mid A) \wedge(D \mid C)$ (resp., by $\overline{(B \mid A) \wedge(D \mid C)}$ ), and the iterated conditional $(A \rightarrow B) \rightarrow(C \rightarrow D)$ is interpreted by $(D \mid C) \mid(B \mid A)$. Moreover, we define validity of connexive principles within Approach 2.

Definition 6. A connexive principle is valid if and only if the associated conditional random quantity is constant and equal to 1.

We now check the validity of the connexive principles in Table 1 according to Definition 6.

Aristotle's Thesis (AT): $\sim(\sim A \rightarrow A)$. We interpret the principle $\sim(\sim A \rightarrow A)$ by the negation of the conditional event $A \mid \bar{A}$, that is by $\overline{A \mid \bar{A}}$, where $\bar{A} \neq \varnothing$. Then, based on equations (5) and (3), it follows that $\overline{A \mid \bar{A}}=1-A|\bar{A}=\bar{A}| \bar{A}=1$. Therefore, (AT) is valid because the conditional random quantity $\overline{A \mid \bar{A}}$, which also coincides with the conditional event $\bar{A} \mid \bar{A}$, is constant and equal to 1 . 
Aristotle's Thesis' $\left(A T^{\prime}\right): \sim(A \rightarrow \sim A)$. We interpret the principle $\sim(A \rightarrow \sim A)$ by the negation of the conditional event $\bar{A} \mid A$, that is by $\bar{A} \mid A$, where $A \neq \varnothing$. Like in (AT), it holds that $\bar{A}|A=1-\bar{A}| A=A \mid A=1$, which validates (AT '). Notice that, (AT ${ }^{\prime}$ ) also follows from (AT) when $A$ is replaced by $\bar{A}$ (of course $\overline{\bar{A}}=A$ ).

Abelard's Thesis $(A B): \sim((A \rightarrow B) \wedge(A \rightarrow \sim B))$. The structure of this principle is formalized by the conditional random quantity $\overline{(B \mid A) \wedge(\bar{B} \mid A)}$, where $A \neq \varnothing$. We observe that $(B \mid A) \wedge(\bar{B} \mid A)=(B \wedge \bar{B})|A=\varnothing| A$. Then, $\overline{(B \mid A) \wedge(\bar{B} \mid A)}=\overline{\varnothing \mid A}=\bar{\varnothing}|A=\Omega| A=1$. Therefore, $(\mathrm{AB})$ is valid.

Aristotle's Second Thesis $(A S): \sim((A \rightarrow B) \wedge(\sim A \rightarrow B))$. The structure of this principle is formalized by the random quantity $\overline{(B \mid A) \wedge(B \mid \bar{A})}$, where $A \neq \varnothing$ and $\bar{A} \neq \varnothing$. By setting $p(B \mid A)=x$ and $p(B \mid \bar{A})=y$, it follows that [19]23]

$$
(B \mid A) \wedge(B \mid \bar{A})=(B \mid A) \cdot(B \mid \bar{A})= \begin{cases}0, & \text { if } A \bar{B} \vee \bar{A} \bar{B} \text { is true } \\ y, & \text { if } A B \text { is true, } \\ x, & \text { if } \bar{A} B \text { is true. }\end{cases}
$$

Then, $\overline{(B \mid A) \wedge(B \mid \bar{A})}=1-(B \mid A) \wedge(B \mid \bar{A})=1-(y A B+x \bar{A} B)$, which is not constant and can therefore not necessarily be equal to 1 . In particular, by choosing the coherent assessment $x=y=1$, it follows that $\overline{(B \mid A) \wedge(B \mid \bar{A})}=1-A B-\bar{A} B=1-B=\bar{B}$, which is not necessarily equal to 1 as it could be either 1 or 0 , according to whether $\bar{B}$ is true or false, respectively. Therefore, (AS) is not valid. Moreover, by setting $\mathbb{P}[(B \mid A) \wedge(B \mid \bar{A})]=\mu$, it holds that $\mu=y p(A B)+x p(\bar{A} B)=y p(B \mid A) p(A)+x p(B \mid \bar{A}) p(\bar{A})=x y p(A)+x y p(\bar{A})=x y$. Then, $\mathbb{P}[\overline{(B \mid A) \wedge(B \mid \bar{A})}]=1-x y$. We also observe that, in the special case where $x=y=0$, it follows that $\overline{(B \mid A) \wedge(B \mid \bar{A})}=1$.

Boethius' Thesis (BT): $(A \rightarrow B) \rightarrow \sim(A \rightarrow \sim B)$. This principle is formalized by the iterated conditional $(\overline{\bar{B} \mid A}) \mid(B \mid A)$, with $A B \neq \varnothing$. We recall that $(\overline{\bar{B} \mid A})=$ $B \mid A$. Then $(\bar{B} \mid A)|(B \mid A)=(B \mid A)|(B \mid A)$. Moreover, by setting $p(B \mid A)=x$ and $\mathbb{P}[(B \mid A) \mid(B \mid A)]=\mu$, it holds that

$$
\begin{aligned}
& (B \mid A) \mid(B \mid A)=(B \mid A) \wedge(B \mid A)+\mu(1-B \mid A)=(B \mid A)+\mu(1-B \mid A)= \\
& = \begin{cases}1, & \text { if } A B \text { is true, } \\
\mu, & \text { if } A \bar{B}, \text { is true, } \\
x+\mu(1-x), & \text { if } \bar{A} \text { is true. }\end{cases}
\end{aligned}
$$

By linearity of prevision it holds that $\mu=x+\mu(1-x)$. Then,

$$
(B \mid A) \mid(B \mid A)=(B \mid A)+\mu(1-B \mid A) \begin{cases}1, & \text { if } A B \text { is true, } \\ \mu, & \text { if } \bar{A} \vee \bar{B} \text { is true. }\end{cases}
$$

Then, by coherence it must be that $\mu=1$ and hence ([22, Remark 2], see also [27, Section 3.2])

$$
(B \mid A) \mid(B \mid A)=1 .
$$

Therefore $(\overline{\bar{B} \mid A}) \mid(B \mid A)$ is constant and equal to 1 and hence (BT) is valid. 
Boethius' Thesis' $\left(B T^{\prime}\right):(A \rightarrow \sim B) \rightarrow \sim(A \rightarrow B)$. This principle is formalized by the iterated conditional $(\overline{B \mid A}) \mid(\bar{B} \mid A)$, where $A \bar{B} \neq \varnothing$. By observing that $\overline{B \mid A}=\bar{B} \mid A$, it follows that $(\overline{B \mid A})|(\bar{B} \mid A)=(\bar{B} \mid A)|(\bar{B} \mid A)$ which is constant and equal to 1 because of (7). Therefore, $\left(\mathrm{BT}^{\prime}\right)$ is valid.

Reversed Boethius' Thesis (RBT): $\sim(A \rightarrow \sim B) \rightarrow(A \rightarrow B)$. This principle is formalized by the iterated conditional $(B \mid A) \mid(\overline{\bar{B} \mid A})$, where $A B \neq \varnothing$. As $(\overline{\bar{B} \mid A})=B \mid A$, it follows from (7) that $(B \mid A)|(\overline{\bar{B} \mid A})=(B \mid A)|(B \mid A)=1$. Therefore, (RBT) is valid.

Reversed Boethius' Thesis' $\left(R B T^{\prime}\right): \sim(A \rightarrow B) \rightarrow(A \rightarrow \sim B)$. This principle is formalized by the iterated conditional $(\bar{B} \mid A) \mid(\overline{B \mid A})$, where $A \bar{B} \neq \varnothing$. As $(\overline{B \mid A})=\bar{B} \mid A$, it follows from (7) that $(\bar{B} \mid A)|(\overline{B \mid A})=(\bar{B} \mid A)|(\bar{B} \mid A)=1$. Therefore $\left(\mathrm{RBT}^{\prime}\right)$ is validated.

Boethius Variation (B3): $(A \rightarrow B) \rightarrow \sim(\sim A \rightarrow B)$. This principle is formalized by the iterated conditional $(\overline{B \mid \bar{A}}) \mid(B \mid A)$, where $A B \neq \varnothing$. We observe that $(\bar{B} \mid \bar{A})|(B \mid A)=(\bar{B} \mid \bar{A})|(B \mid A)$, because $\overline{B \mid \bar{A}}=\bar{B} \mid \bar{A}$. By setting $p(B \mid A)=x$, $p(\bar{B} \mid \bar{A})=y$, and $\mathbb{P}[(\bar{B} \mid \bar{A}) \mid(B \mid A)]=\mu$, it holds that

$$
(\bar{B} \mid \bar{A}) \mid(B \mid A)=(\bar{B} \mid \bar{A}) \wedge(B \mid A)+\mu(1-B \mid A)= \begin{cases}y, & \text { if } A B \text { is true } \\ \mu, & \text { if } A \bar{B} \text { is true } \\ \mu(1-x), & \text { if } \bar{A} B \text { is true } \\ x+\mu(1-x), & \text { if } \bar{A} \bar{B} \text { is true }\end{cases}
$$

which is not constant and can therefore not necessarily be equal to 1 . For example, if we choose the coherent assessment $x=y=1$, it follows that

$$
(\bar{B} \mid \bar{A}) \mid(B \mid A)=(\bar{B} \mid \bar{A}) \wedge(B \mid A)+\mu(1-B \mid A)= \begin{cases}1, & \text { if } A B \text { is true } \\ \mu, & \text { if } A \bar{B} \text { is true } \\ 0, & \text { if } \bar{A} B \text { is true } \\ 1, & \text { if } \bar{A} \bar{B} \text { is true }\end{cases}
$$

which is not constant and equal to 1 . Therefore, (B3) is not valid.

Boethius Variation (B4): $(\sim A \rightarrow B) \rightarrow \sim(A \rightarrow B)$. This principle is formalized by the iterated conditional $(\overline{B \mid A}) \mid(B \mid \bar{A})$, where $\bar{A} B \neq \varnothing$. We observe that $(\overline{B \mid A}) \mid(B \mid \bar{A})$ is not constant and not necessarily equal to 1 because it is equivalent to (B3) when $A$ is replaced by $\bar{A}$. Therefore, (B4) is not valid.

Connexive principles and their interpretation in terms of compound or iterated conditionals are illustrated in Table 4.

\section{Concluding Remarks}

We presented two approaches to investigate connexive principles. Connexivity is interpreted by in terms of probabilistic constraints on conditional events (in the sense of defaults, or negated defaults) in Approach 1. Within this approach we showed that the connexive principles $(\mathrm{AT}),\left(\mathrm{AT}^{\prime}\right),(\mathrm{AB}),(\mathrm{BT})$, and $\left(\mathrm{BT}^{\prime}\right)$ 


\begin{tabular}{|c|c|c|c|c|}
\hline Name & Connexive principle & Interpretation & Value & Validity \\
\hline (AT) & $\sim(\sim A \rightarrow A)$ & $\overline{A \mid \bar{A}}$ & $=1$ & yes \\
\hline$\left(\mathrm{AT}^{\prime}\right)$ & $\sim(A \rightarrow \sim A)$ & $\bar{A} \mid A$ & $=1$ & yes \\
\hline$(\mathrm{AB})$ & $\sim((A \rightarrow B) \wedge(A \rightarrow \sim B))$ & $\overline{(B \mid A) \wedge(\bar{B} \mid A)}$ & $=1$ & yes \\
\hline (AS) & $\sim((A \rightarrow B) \wedge(\sim A \rightarrow B))$ & $\overline{(B \mid A) \wedge(B \mid \bar{A})}$ & $\neq 1$ & no \\
\hline$(\mathrm{BT})$ & $(A \rightarrow B) \rightarrow \sim(A \rightarrow \sim B)$ & $\overline{(\bar{B} \mid A)} \mid(B \mid A)$ & $=1$ & yes \\
\hline$\left(\mathrm{BT}^{\prime}\right)$ & $(A \rightarrow \sim B) \rightarrow \sim(A \rightarrow B)$ & $\overline{(B \mid A)} \mid(\bar{B} \mid A)$ & $=1$ & yes \\
\hline$(\mathrm{RBT})$ & $\sim(A \rightarrow \sim B) \rightarrow(A \rightarrow B)$ & $(B \mid A) \mid \overline{(\bar{B} \mid A)}$ & $=1$ & yes \\
\hline$\left(\mathrm{RBT}^{\prime}\right)$ & $\sim(A \rightarrow B) \rightarrow(A \rightarrow \sim B)$ & $(\bar{B} \mid A) \mid \overline{(B \mid A)}$ & & yes \\
\hline (B3) & $(A \rightarrow B) \rightarrow \sim(\sim A \rightarrow B)$ & $\overline{(B \mid \bar{A})} \mid(B \mid A)$ & $\neq 1$ & no \\
\hline (B4) & $(\sim A \rightarrow B) \rightarrow \sim(A \rightarrow B)$ & $\overline{(B \mid A)} \mid(B \mid \bar{A})$ & $\neq 1$ & no \\
\hline
\end{tabular}

Table 4: Connexive principles in the framework of compounds of conditionals and iterated conditionals (Approach 2). Value denotes whether the conditional random quantity is constant and equal to 1 .

are valid, whereas (AS), (RBT), $\left(\mathrm{RBT}^{\prime}\right),(\mathrm{B} 3)$, and (B4) are not valid (see Table 3). In Approach 2 connexivity is interpreted by constraints on compounds of conditionals and iterated conditionals. Here, we demonstrated that, like in Approach 1, (AT), (AT'), (AB), (BT), and $\left(\mathrm{BT}^{\prime}\right)$ are valid, whereas (AS), (B3), and (B4) are not valid. Contrary to Approach 1, (RBT) and $\left(\mathrm{RBT}^{\prime}\right)$ are valid in Approach 2 (see Table 4).

Approach 1 is characterized by employing concepts from coherence-based probability theory and probabilistic interpretations of defaults and negated defaults. Conditionals, interpreted as defaults, are negated by the wide scope negation. We gave two notions of validity, namely for non-iterated and iterated connexive principles, respectively. Approach 2 allows for dealing with logical operations on conditional events and avoids (see, e.g., [51]) the well known Lewis' triviality results (see, e.g., [35]). It therefore offers a more unified approach to connexive principles, which is reflected by a unique definition of validity for both, iterated and non-iterated connexive principles. Moreover, Approach 2 negates conditionals by the narrow scope negation. Thus, validity depends on how conditionals and negation are defined.

One might wonder why neither of the two approaches validates all connexive principles. Of course, we have shown by proofs why, for instance, (AS) is not valid in both approaches. Apart from the insight obtained from our proofs, this is not surprising since also not all connexive principles are valid in all systems of connexive logic. Moreover, some rules which are valid in classical logic (e.g., transitivity, contraposition, and premise strengthening) are not valid in probability logic, while, for example, the rules of the basic nonmonotonic System P are valid within coherence-based probability logic (915]16|24|17).

We have shown that coherence-based probability logic offers a rich language to investigate the validity of various connexive principles. Future work will be devoted to investigations on other intuitively plausible logical principles contained in alternative and non-classical logics.

Acknowledgments. Thanks to four anonymous reviewers for useful comments. 


\section{References}

1. Adams, E.W.: The logic of conditionals. An application of probability to deduction. Reidel, Dordrecht (1975)

2. Berti, P., Regazzini, E., Rigo, P.: Well calibrated, coherent forecasting systems. Theory of Probability \& Its Applications 42(1), 82-102 (1998). https://doi.org/https://doi.org/10.1137/S0040585X97975988

3. Biazzo, V., Gilio, A.: A generalization of the fundamental theorem of de Finetti for imprecise conditional probability assessments. International Journal of Approximate Reasoning 24(2-3), 251-272 (2000)

4. Biazzo, V., Gilio, A., Lukasiewicz, T., Sanfilippo, G.: Probabilistic logic under coherence, model-theoretic probabilistic logic, and default reasoning in System P. Journal of Applied Non-Classical Logics 12(2), 189-213 (2002)

5. Calabrese, P.: Logic and Conditional Probability: A Synthesis. College Publications (2017)

6. Capotorti, A., Lad, F., Sanfilippo, G.: Reassessing accuracy rates of median decisions. The American Statistician 61(2), 132-138 (2007)

7. Ciucci, D., Dubois, D.: Relationships between Connectives in Three-Valued Logics. In: Advances on Computational Intelligence, CCIS, vol. 297, pp. 633-642. Springer (2012)

8. Ciucci, D., Dubois, D.: A map of dependencies among three-valued logics. Information Sciences 250, 162 - 177 (2013). https://doi.org/10.1016/j.ins.2013.06.040

9. Coletti, G., Scozzafava, R.: Probabilistic logic in a coherent setting. Kluwer, Dordrecht (2002)

10. Coletti, G., Scozzafava, R., Vantaggi, B.: Possibilistic and probabilistic logic under coherence: Default reasoning and System P. Mathematica Slovaca 65(4), 863-890 (2015)

11. de Finetti, B.: Sul significato soggettivo della probabilitá. Fundamenta Mathematicae 17, 298-329 (1931)

12. de Finetti, B.: Theory of probability, vol. 1, 2. John Wiley \& Sons, Chichester $(1970 / 1974)$

13. Flaminio, T., Godo, L., Hosni, H.: Boolean algebras of conditionals, probability and logic. Artificial Intelligence 286, 103347 (2020). https://doi.org/10.1016/j.artint.2020.103347

14. Freund, M., Lehmann, D., Morris, P.: Rationality, transitivity, and contraposition. Artificial Intelligence 52(2), 191-203 (1991)

15. Gilio, A.: Probabilistic reasoning under coherence in System P. Annals of Mathematics and Artificial Intelligence 34, 5-34 (2002)

16. Gilio, A., Pfeifer, N., Sanfilippo, G.: Transitivity in coherencebased probability logic. Journal of Applied Logic 14, 46-64 (2016). https://doi.org/http://dx.doi.org/10.1016/j.jal.2015.09.012

17. Gilio, A., Pfeifer, N., Sanfilippo, G.: Probabilistic entailment and iterated conditionals. In: Elqayam, S., Douven, I., Evans, J.S.B.T., Cruz, N. (eds.) Logic and Uncertainty in the Human Mind: A Tribute to David E. Over, pp. 71-101. Routledge, Oxon (2020). https://doi.org/10.4324/9781315111902-6

18. Gilio, A., Sanfilippo, G.: Conditional random quantities and iterated conditioning in the setting of coherence. In: van der Gaag, L.C. (ed.) ECSQARU 2013, LNCS, vol. 7958, pp. 218-229. Springer, Berlin, Heidelberg (2013)

19. Gilio, A., Sanfilippo, G.: Conjunction, disjunction and iterated conditioning of conditional events. In: Kruse, R., Berthold, M.R., Moewes, C., Gil, M.A., Grzegorzewski, P., Hryniewicz, O. (eds.) Synergies of Soft Computing and Statistics for 
Intelligent Data Analysis, AISC, vol. 190, pp. 399-407. Springer, Berlin, Heidelberg (2013)

20. Gilio, A., Sanfilippo, G.: Probabilistic entailment in the setting of coherence: The role of quasi conjunction and inclusion relation. International Journal of Approximate Reasoning 54(4), 513-525 (2013)

21. Gilio, A., Sanfilippo, G.: Quasi conjunction, quasi disjunction, t-norms and t-conorms: Probabilistic aspects. Information Sciences 245, 146-167 (2013). https://doi.org/10.1016/j.ins.2013.03.019

22. Gilio, A., Sanfilippo, G.: Conditional random quantities and compounds of conditionals. Studia Logica 102(4), 709-729 (2014)

23. Gilio, A., Sanfilippo, G.: Conjunction of conditional events and t-norms. In: KernIsberner, G., Ognajović, Z. (eds.) Symbolic and Quantitative Approaches to Reasoning with Uncertainty (ECSQARU 2019), Lecture Notes in Computer Science, vol. 11726, pp. 199-211. Springer (2019)

24. Gilio, A., Sanfilippo, G.: Generalized logical operations among conditional events. Applied Intelligence 49(1), 79-102 (2019)

25. Gilio, A., Sanfilippo, G.: Algebraic aspects and coherence conditions for conjoined and disjoined conditionals. International Journal of Approximate Reasoning 126, 98-123 (2020)

26. Gilio, A., Sanfilippo, G.: Compound conditionals, Fréchet-Hoeffding bounds, and Frank t-norms. International Journal of Approximate Reasoning 136, 168-200 (2021). https://doi.org/https://doi.org/10.1016/j.ijar.2021.06.006

27. Gilio, A., Sanfilippo, G.: On compound and iterated conditionals. Argumenta (To Appear)

28. Goodman, I.R., Nguyen, H.T., Walker, E.A.: Conditional Inference and Logic for Intelligent Systems: A Theory of Measure-Free Conditioning. North-Holland (1991), www.dtic.mil/dtic/tr/fulltext/u2/a241568.pdf

29. Holzer, S.: On coherence and conditional prevision. Bollettino dell'Unione Matematica Italiana 4(6), 441-460 (1985)

30. Horn, L.R.: A natural history of negation. CSLI Publications, Stanford (2001)

31. Kaufmann, S.: Conditionals right and left: Probabilities for the whole family. Journal of Philosophical Logic 38, 1-53 (2009)

32. Kraus, S., Lehmann, D., Magidor, M.: Nonmonotonic reasoning, preferential models and cumulative logics. Artificial Intelligence 44, 167-207 (1990)

33. Lad, F.: Operational subjective statistical methods: A mathematical, philosophical, and historical introduction. Wiley, New York (1996)

34. Lenzen, W.: A critical examination of the historical origins of connexive logic. History and Philosophy of Logic 41(1), 16-35 (2020)

35. Lewis, D.: Probabilities of conditionals and conditional probabilities. Philosophical Review 85, 297-315 (1976)

36. McCall, S.: A history of connexivity. In: Gabbay, D.M., Pelletier, F.J., Woods, J. (eds.) Handbook of the history of logic, vol. 11 (Logic: a history of its central concepts). Elsevier, Amsterdam (2012)

37. McGee, V.: Conditional probabilities and compounds of conditionals. Philosophical Review 98, 485-541 (1989)

38. Pearl, J.: Probabilistic semantics for nonmonotonic reasoning: A survey. In: Shafer, G., Pearl, J. (eds.) Readings in Uncertain Reasoning, pp. 699-711. Morgan Kaufmann, San Mateo (1990)

39. Pfeifer, N.: Experiments on Aristotle's Thesis: Towards an experimental philosophy of conditionals. The Monist 95(2), 223-240 (2012) 
40. Pfeifer, N.: Probability logic. In: Knauff, M., Spohn, W. (eds.) Handbook of Rationality. MIT Press, Cambridge (in press)

41. Pfeifer, N., Kleiter, G.D.: Coherence and nonmonotonicity in human reasoning. Synthese 146(1-2), 93-109 (2005)

42. Pfeifer, N., Kleiter, G.D.: Framing human inference by coherence based probability logic. Journal of Applied Logic 7(2), 206-217 (2009)

43. Pfeifer, N., Kleiter, G.D.: The conditional in mental probability logic. In: Oaksford, M., Chater, N. (eds.) Cognition and conditionals: Probability and logic in human thought, pp. 153-173. Oxford University Press, Oxford (2010)

44. Pfeifer, N., Sanfilippo, G.: Probabilistic squares and hexagons of opposition under coherence. International Journal of Approximate Reasoning 88, 282-294 (2017)

45. Pfeifer, N., Sanfilippo, G.: Probabilistic semantics for categorical syllogisms of Figure II. In: Ciucci, D., Pasi, G., Vantaggi, B. (eds.) Scalable Uncertainty Management: 12th International Conference, SUM 2018, Lecture Notes in Computer Science, vol. 11142, pp. 196-211. Springer International Publishing, Cham (2018)

46. Pfeifer, N., Sanfilippo, G.: Probability propagation in selected Aristotelian syllogisms. In: Kern-Isberner, G., Ognajović, Z. (eds.) Symbolic and Quantitative Approaches to Reasoning with Uncertainty (ECSQARU 2019), Lecture Notes in Computer Science, vol. 11726, pp. 419-431. Springer (2019)

47. Pfeifer, N., Tulkki, L.: Conditionals, counterfactuals, and rational reasoning. An experimental study on basic principles. Minds and Machines 27(1), 119-165 (2017)

48. Ramsey, F.P.: General propositions and causality (1929). In: Mellor, D.H. (ed.) Philosophical Papers by F. P. Ramsey, pp. 145-163. Cambridge University Press, Cambridge (1929/1994)

49. Regazzini, E.: Finitely additive conditional probabilities. Rendiconti del Seminario Matematico e Fisico di Milano 55, 69-89 (1985)

50. Sanfilippo, G.: Lower and upper probability bounds for some conjunctions of two conditional events. In: SUM 2018, LNCS, vol. 11142, pp. 260-275. Springer, Cham (2018)

51. Sanfilippo, G., Gilio, A., Over, D.E., Pfeifer, N.: Probabilities of conditionals and previsions of iterated conditionals. International Journal of Approximate Reasoning 121, 150-173 (2020)

52. Walley, P., Pelessoni, R., Vicig, P.: Direct algorithms for checking consistency and making inferences from conditional probability assessments. Journal of Statistical Planning and Inference 126(1), 119-151 (2004)

53. Wansing, H.: Connexive logic. In: Zalta, E.N. (ed.) The Stanford Encyclopedia of Philosophy. Spring 2020 edn. (2020) 